\author{
Proceedings of the $9^{\text {th }}$ International Conference on Applied Informatics \\ Eger, Hungary, January 29-February 1, 2014. Vol. 2. pp. 139-144 \\ doi: 10.14794/ICAI.9.2014.2.139
}

\title{
The effect of dynamic active-inactive agents on spreading phenomena
}

\author{
Gergely Kocsis, Imre Varga \\ University of Debrecen, Faculty of Informatics \\ \{kocsis.gergely, varga.imre\}@inf .unideb.hu
}

\begin{abstract}
In this work a novel model of spreading processes is presented. The model is an upgrade of a previously introduced model of information spreading [1]. Here we take into account that agents of the system are not always active during the spreading and information can flow only through those that are currently active. In order to suit our work to other findings related to spreading phenomena we describe our model using the classical terminology of disease spreading. Namely our model can be viewed as an SRS/SIRI model of disease spreading [4]. Based on this model we carried out computer simulations showing that the activity of agents highly affect the process. The results of the work can be used to qualitatively predict what would be the effect if the activity of agents change in a social system e.g. in a social network.
\end{abstract}

Keywords: cellular automata, spreading, active nodes

$M S C: 68 \mathrm{U} 20,91 \mathrm{D} 10,91 \mathrm{D} 30$

\section{Introduction}

However social spreading phenomena is being investigated for a couple of decades $[2,3]$, the information society of the $X X I$ st century keeps opening new aspects of it making it a permanently relevant and actual topic. One special case of spreading phenomena is the spreading of information on social networks. The relevance of this topic can be easily understood knowing that the related findings can be easily used to tune online marketing strategies or, from the other point of view, for anti-spam applications. Previously we proposed a model of information spreading where we focused on the competition of two different information channels. In this work we update this model, and reform it so that it uses the usual terminology of disease spreading models. Namely based on our previous experiences, we simplify the model in some points, while in the mean time we introduce a new parameter to 


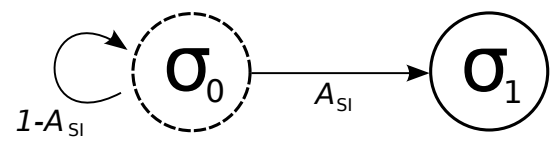

Figure 1: State-change rules of the SI model. Susceptible agents $\left(\sigma_{0}\right)$ can get infected with probability $A_{S I}$. From this naturally the probability to stay in $\sigma_{0}$ is $\left(1-A_{S I}\right)$. An agent that is once infected can not change its state.

describe activity of agents of the system. Using the model we would like to find out how the activity of agents affect the spreading. In order to get an insight of the work of our model we run computer simulations based on square lattice topology.

\section{Possible models of information spreading}

\subsection{The SI model}

The very first model that can be taken into account, when one models information spreading phenomena is the SI (susceptible-infected) model of epidemic spreading [4]. Though this model is about 90 years old, it is a very good starting point because of its simplicity. In the model we introduce agents that can be in two different states. Agents represent actors of a social system and their states describe that e.g. in our case they are informed or not. The traditional notation of these states are $S$ and $I$ but in order to be able to preserve the same notations during our different models lets name them respectively $\sigma_{0}$ and $\sigma_{1}$. The evolution of this model is based only on one rule that describes the conditions of an agent to change its state from $\sigma_{0}$ to $\sigma_{1}$. Namely agents can change their state in each discrete steps of time with a probability $A_{S I}$, where $A_{S I}$ depends on the amount of infected neighbors. The basic rule of the model is presented on Figure 1.

Historically in the case of this model the assumption is used to be made that all agents are connected, however in simulations it is also possible to use different topologies. However this model is a very good starting point we found that if we would like to model for example information spreading in a more realistic way, we have to add some extra parameters.

\subsection{The two channel SI model}

As we presented in a previous work of ours information spreading in social systems is mainly driven by two common channels. $i$.) There is an inner channel based on the communication of connected agents, $i i$.) and usually there is also an outer source of information that acts as a constant driving force of the system. Taking into account the effect of these two channels we presented a modification of the SI model, where the transition rule from $\sigma_{0}$ to $\sigma_{1}$ (i.e. from $\mathrm{S}$ to I) is based on the amount of information received by a given agent. More precisely if the sensitivity of 


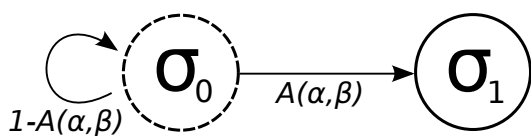

Figure 2: State-change rules the tho channel SI model. Compared to the SI model the only change is in the probability $A$.

agents to the inner and to the outer information channels are $\alpha$ and $\beta$ respectively, agent $i$ gets informed at time $t$ with probability

$$
A(\alpha, \beta)=1-\exp \left(-\left(\alpha S_{i} \sum_{j=1}^{n_{i}}\left(1-S_{j}\right)+\beta S_{i}\right)\right),
$$

where

$$
S_{i}= \begin{cases}1, & \text { if agent } i \text { is uninformed } \\ 0, & \text { if agent } i \text { is informed }\end{cases}
$$

One may note that compared to the original model presented in [1] the above description is simplified in many aspects. However note also that according to the results presented in the referred paper, making these simplifications does not affect the qualitative behavior of the model. Nevertheless since we would like to examine the effect of dynamic activity, it is better to start from a more simple model. The basic rule of the model is presented on Figure 2.

\subsection{The SRS/SIRI model}

In our final model the actors of the social system are again represented by interacting agents put on a given network topology. Just as in the case of the two channel SI model, agents are described by their states, where the state of an agent depends on two values. Namely $S_{i} \in\{0,1\}$ that tells if an agent is informed or not and $R_{i} \in\{0,1\}$ that tells if an agent is inactive or active respectively. We keep the sensitivities $\alpha$ and $\beta$ from the two channel SI model, while we introduce a third system wide parameter as well. Namely the probabilistic value $\gamma$ tells how often agents change their $R$ value from 1 to 0 . For all three values $\alpha, \beta, \gamma \in[0,1] \in \mathbb{R}$.

From the above description it comes that agents have 4 different states to be in. $\sigma_{0}$ (active-uninformed), $\sigma_{1}$ (inactive-uninformed), $\sigma_{2}$ (active-informed) and $\sigma_{3}$ (inactive-informed). In terms of the classical notation $\sigma_{0}$ is the susceptible state, $\sigma_{1}$ and $\sigma_{3}$ are the removed states while $\sigma_{2}$ is the state of infected agents. The acronym of the model comes from the possible state change series $(S \rightarrow R \rightarrow S$, and $\mathrm{S} \rightarrow \mathrm{I} \rightarrow \mathrm{R} \rightarrow \mathrm{I})$.

Figure 3. presents the possible state changes of agents. In short, agents get or stay inactive with probability $\gamma$ and stay or change active with probability $1-\gamma$. The probability of changing from $\sigma_{0}$ to $\sigma_{2}$ thus getting informed is a little bit more sophisticated. Namely based on the two channel SI model this probability can be cast to the form 


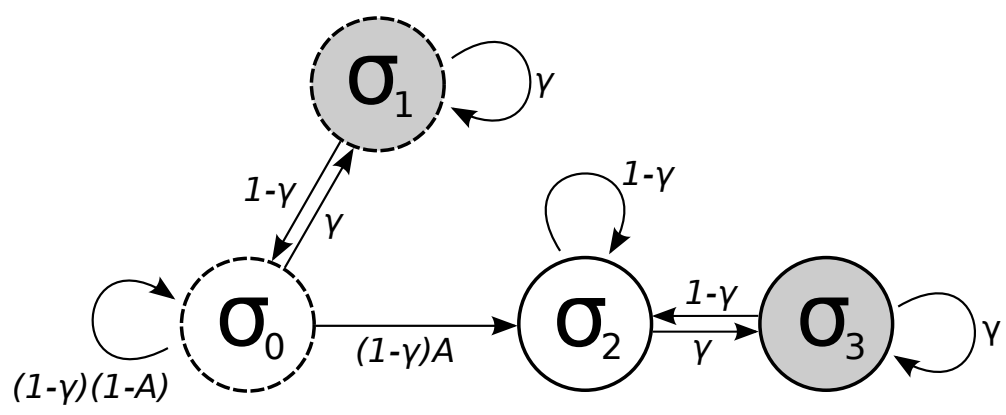

Figure 3: State-change rules of the SRS/SIRI model. Dashed circles mark uninformed agents. Continuous lined circles are for informed agents. Grey filling indicates inactive states. With probability $\gamma$ agents go/stay inactive and with probability $1-\gamma$ they go/stay active. The rule of getting informed is based on the two channel SI model.

$$
A=1-\exp \left(-\left(\alpha S_{i} R_{i} \sum_{j=1}^{n_{i}}\left(\left(1-S_{j}\right) R_{j}\right)+\beta S_{i} R_{i}\right)\right),
$$

where $n_{i}$ is the number of neighbors of agent $i$. Note that however all agents have the same values for $\alpha$ and $\beta$ only active agents can spread and receive information because of the presence of $R$.

\section{Results of computer simulations}

In order to get an insight to the behavior of our system the time evolution of the system was investigated through computer simulations for different parameters. The values of the parameters were set to the values proven to be correct in [1] thus $\alpha=10^{-2}$ and $\beta=10^{-4}$, while the system size was $N=10^{6}$. We ran our simulations on square lattices with periodic boundary condition. The results of a small sample run on a system of $N=10^{4}$ agents with $\gamma=0.5$ are presented on Figure 4. According to the figure at first some agents get informed due to the outer information channel. From these informed agents small clusters of informed agents start to grow, while after a while a system wide so called giant cluster appears. Comparing these qualitative finding with the results found in the case of the two channel SI model, we find that however the introduction of dynamic activity does not have a qualitative effect it slows down the evolution of the system.

To understand more this slowing we examined the amount of informed agents in the system during its evolution. Figure 5. shows the results of plotting the percentage of informed agents in the system $\left(\sigma_{2}+\sigma_{3}\right)$ as a function of time for different values of $\gamma$. 


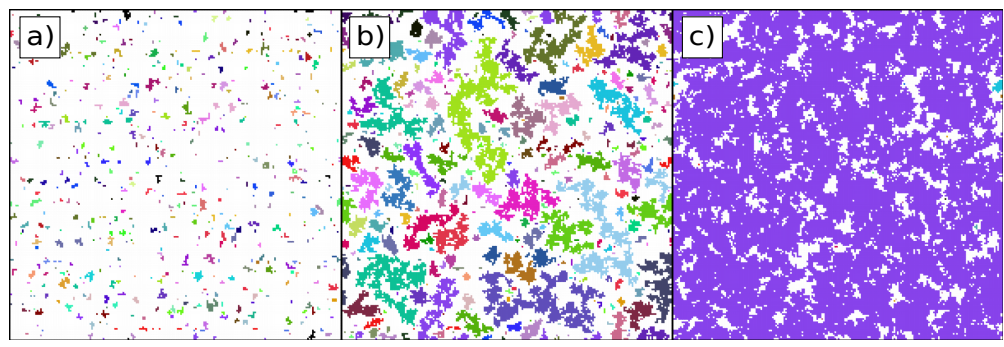

Figure 4: (color online) Snapshots of the system on a square lattice taken at $t=350,800,1250$ for $a, b$ and $c$ respectively. The level of inactivity is $\gamma=0.5$. At the beginning random agents get informed. Later these informed agents spread the information to their active neighbors, while at the end a system wide giant cluster of informed agents appear.

Note that the curves on Figure 5. a) show the well known logistic form that usually appears in the case of diffusion processes. It can also be seen clearly that however the spreading process shows the same fashion regardless of the value of $\gamma$ the time needed to reach the state where $95 \%$ the agents are informed $t_{\langle S\rangle=0.95}$ is not a linear function of $\gamma$. Figure 5. b) also shows that this dependence is faster than exponential. The reason why we chose to examine $t_{\langle S\rangle=0.95}$ instead of $t_{\langle S\rangle=1}$ is that the spreading slows down so much close to the homogeneous state that $t_{\langle S\rangle=1} \rightarrow \infty$, however $t_{\langle S\rangle=0.95}$ still fits our needs while the respective value can be reached in a reasonable time.

These results of course are very artificial, because of the regular lattice topology, but they still provide a good first qualitative feeling about the evolution of the system. To bring our results closer to the real world, we need to change the topology of the underlying network topology in the future. Good candidates are rewired lattices [5] and complex networks [6].

\section{Discussion}

In this paper we introduced a novel model of spreading phenomena on social systems where the actors are active only in a given part of their time. The model is based on two previous models of the literature. Namely it uses the terms of the SI model while the logic of it follows the two channel information spreading model. Based on computer simulations we showed that the increased inactivity of agents slows down the spreading however this change does not have a qualitative effect. Since the square lattice topology is far from being realistic, in the future we plan to test the model on other topologies as well such as small-world networks or scale-free networks. Those results may be also compared to real world data, so that one can find a practical use of our work. 
a)

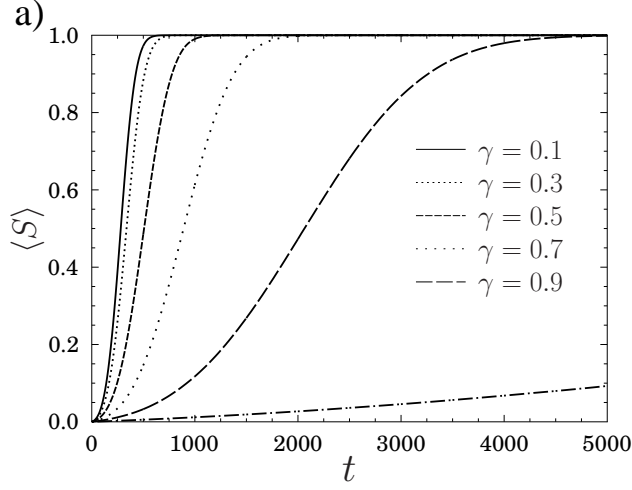

b)

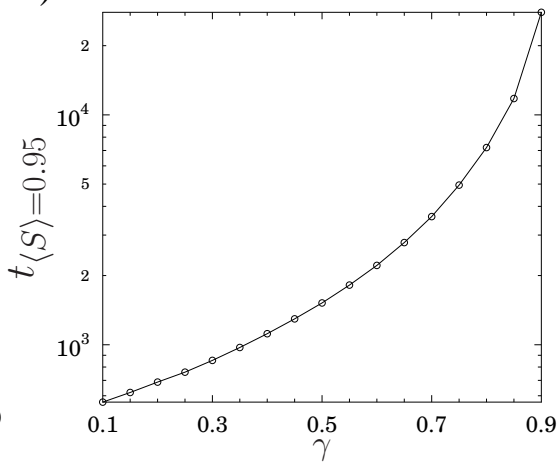

Figure 5: Simulation results on square lattices. a) The ratio of informed agents in the system as a function of time for different levels of inactivity $\gamma$. b) The time needed to reach $95 \%$ homogeneous informed state in the system. The semi-log plot shows that the dependence on $\gamma$ is faster than exponential.

Acknowledgements. The publication was supported by the TÁMOP-4.2.2.C11/1/KONV-2012-0001 project. The project has been supported by the European Union, co-financed by the European Social Fund.

\section{References}

[1] G. Kocsis and F. Kun, Competition of information channels in the spreading of innovations. Physical Review E 84, 2 (2011).

[2] E. M. Rogers, Diffusion of Innovations, Fifth Edition (New York, Free Press, ISBN 0-7432-2209-1) (2003).

[3] W. Weidlich, Sociodynamics: A systematic approach to mathematical modelling in the social sciences, 113-187 (Dover Publications, Mineola, USA, 2000).

[4] M. E. J. Newman, Networks - An introduction. University of Michigan and Santa Fe Institute, Oxford University Press pp. 627-675 (2010).

[5] D. J. Watts and S. H. Strogatz, Collective dynamics of "small-world" networks, Nature, 393, 440-442, (1998).

[6] A. Barabasi and R. Albert, Emergence of scaling in random networks, Science 286 pp. 509512 (1999). 